\title{
Set Target Aggregation of Multiple Mechanical Systems
}

\author{
Ziyang Meng, Tao Yang, Guodong Shi, Dimos V. Dimarogonas, \\ Yiguang Hong, and Karl H. Johansson
}

\begin{abstract}
In this paper, we study the set target aggregation problem of multiple mechanical systems. Each system is modeled by a Lagrangian dynamical equation and observes a convex set as its local target. The objective of the group is to reach an aggregation towards these target sets. We propose a set target aggregation algorithm that is constructed based on each mechanical system's own target sensing and the exchange of its information with local neighbors. With necessary connectivity for both fixed and switching communication topologies, multiple mechanical systems are shown to converge to the intersection of all the local target sets while the vectors of generalized coordinate derivatives are driven to zero. Simulations are given to validate the theoretical results.
\end{abstract}

\section{INTRODUCTION}

A large amount of results have been obtained towards distributed control of multi-agent systems during the last several decades (see e.g., [1]-[3]). The key idea is to realize a collective task for the overall system by using only local information interactions [4]-[6]. Such an algorithm naturally requires that the individual system is equipped with communication units and therefore the analysis on the influence of the communication link failure and communication data loss is important [2], [7]. Also, for the agent dynamics, both continuous-time and discrete-time models were studied and deep understanding was obtained including convergence speed, delay robustness, nonlinear convexity, general linear dynamics and so on [8]-[12].

The single integrator model assumption has been widely used in the literature to derive mathematically beautiful results. On the other hand, in order to satisfy real application requirements, the study on the distributed control of multiple Lagrangian systems has attracted extensive attention recently. Here, a Lagrangian model can be used to describe mechanical systems including autonomous vehicles, mobile robots, robotic manipulators, and rigid bodies [13]-[16]. In particular, the author of [17] proposed distributed modelindependent consensus algorithms for multiple Lagrangian systems in a leaderless setting. The rendezvous algorithm was proposed for multiple nonholonomic vehicles in [18]

Z. Meng, T. Yang, G. Shi, D. V. Dimarogonas, and K. H. Johansson are with ACCESS Linnaeus Centre, School of Electrical Engineering, Royal Institute of Technology, Stockholm 10044, Sweden. Y. Hong is with Key Laboratory of Systems and Control, Institute of Systems Science, Chinese Academy of Sciences, Beijing 100190, China. Email: \{ziyangm, taoyang, guodongs, dimos, kallej\}@kth.se, yghongeiss.ac.cn.

This work has been supported in part by the Knut and Alice Wallenberg Foundation, the Swedish Research Council, and EU HYCON2. An extended version of this work was recently submitted to IEEE Transactions on Control of Network Systems. while finite-time coordinated tracking algorithms were presented in [19] over graphs that are quasi-strongly connected. In order to obtain zero-error coordinated tracking result, a sliding mode based strategy was proposed in [20] for the leader-follower tracking problem. A similar problem was also studied in [21], [22], where continuous control algorithms were proposed. The authors of [23] established containment, group dispersion and group cohesion behaviors for multiple Lagrangian systems, where both the cases of constant and time-varying leaders' velocities were considered. The authors of [24] applied the coordination algorithms to the shape and formation control.

In this paper, we study the set target aggregation problem of multiple mechanical systems driven by Lagrangian dynamical equation and each agent observes a convex set as its local target. We propose a set target aggregation algorithm that is constructed based on each agent's own target sensing and exchange of its information with local neighbors. We show that multiple Lagrangian systems converge to the intersection of all the local target sets while the vectors of generalized coordinate derivatives of all the agents are driven to zero for both cases of fixed communication topology and switching communication topology provided that some necessary connectivity conditions are satisfied.

The remainder of the paper is organized as follows. In Section II, we give some basic notation and definitions on convex analysis, graph theory, and Dini derivatives. We then formulate the problem in Section III. The main results are presented in Sections IV and V for the cases of fixed and switching communication topologies, respectively. The simulations are followed in Section VI and a brief concluding remark is given in Section VII.

\section{Preliminaries}

\section{A. Convex Analysis}

Denote $\|\cdot\|$ the Euclidean norm. For any nonempty set $\mathcal{S} \subseteq \mathbb{R}^{m}$, we use $\|x\|_{\mathcal{S}}=\inf _{y \in \mathcal{S}}\|x-y\|$ to describe the distance between $x \in \mathbb{R}^{m}$ and $\mathcal{S}$. A set $\mathcal{S} \subset \mathbb{R}^{m}$ is called convex if $(1-\zeta) x+\zeta y \in \mathcal{S}$ when $x \in \mathcal{S}, y \in \mathcal{S}$, and $0 \leq \zeta \leq 1$

Let $\mathcal{S}$ be a convex set. The convex projection of any $x \in \mathbb{R}^{m}$ onto $\mathcal{S}$ is denoted by $P_{\mathcal{S}}(x) \in \mathcal{S}$ satisfying $\| x-$ $P_{\mathcal{S}}(x)\|=\| x \|_{\mathcal{S}}$. We also know that $\|x\|_{\mathcal{S}}^{2}$ is continuously differentiable for all $x \in \mathbb{R}^{m}$, and its gradient can be explicitly obtained by [25]:

$$
\nabla\|x\|_{\mathcal{S}}^{2}=2\left(x-P_{\mathcal{S}}(x)\right) .
$$


Also, it is trivial to see that

$$
\left(P_{\mathcal{S}}(x)-x\right)^{\mathrm{T}}\left(P_{\mathcal{S}}(x)-y\right) \leq 0, \quad \forall y \in \mathcal{S},
$$

and

$$
\left\|P_{\mathcal{S}}(x)-P_{\mathcal{S}}(y)\right\| \leq\|x-y\|, \quad \forall x, y \in \mathbb{R}^{m} .
$$

\section{B. Graph theory}

An undirected graph $\mathcal{G}$ consists of a pair $(\mathcal{V}, \mathcal{E})$, where $\mathcal{V}=\{1,2, \ldots, n\}$ is a finite, nonempty set of nodes and $\mathcal{E} \subseteq \mathcal{V} \times \mathcal{V}$ is a set of unordered pairs of nodes. An arc $(j, i) \in \mathcal{E}$ denotes that node $i$ can obtain information from node $j$. An undirected graph $\mathcal{G}$ is defined such that for any two nodes $i$ and $j,(j, i) \in \mathcal{E}$ if and only if $(i, j) \in \mathcal{E}$. All neighbors of node $i$ are denoted $\mathcal{N}_{i}:=\{j:(j, i) \in \mathcal{E}\}$. A path is a sequence of arcs of the form $(i, j),(j, k), \ldots$ An undirected graph $\mathcal{G}$ is said to be connected if each node has an undirected path to any other node.

The weighted adjacency matrix $\mathcal{A}=\left[a_{i j}\right] \in \mathbb{R}^{n \times n}$ associated with the graph $\mathcal{G}$ is defined such that $a_{i j}$ is positive if $(j, i) \in \mathcal{E}$ and $a_{i j}=0$ otherwise. We also assume that $a_{i j}=a_{j i}$, for all $i, j \in \mathcal{V}$ for the undirected graph in this paper. The weighted Laplacian matrix $\mathcal{L}=\left[l_{i j}\right] \in \mathbb{R}^{n \times n}$ associated with $\mathcal{A}$ is defined as $l_{i i}=\sum_{j \neq i} a_{i j}$ and $l_{i j}=$ $-a_{i j}$, where $i \neq j$.

\section{Dini derivatives and Matrosov's theorem}

Let $D^{+} V(x(t), t)$ be the upper Dini derivative of $V(x(t), t)$ with respect to $t$, i.e.,

$$
D^{+} V(x, t)=\limsup _{\tau \rightarrow 0^{+}} \frac{V(x(t+\tau), t+\tau)-V(x(t), t)}{\tau} .
$$

The following lemma will be used for our analysis later.

Lemma 1. ( [26]) Suppose for each $i \in \mathcal{V}, V_{i}: \mathbb{R}^{m} \times$ $\mathbb{R} \rightarrow \mathbb{R}$ is continuously differentiable. Let $V(x, t)=$ $\max _{i \in \mathcal{V}} V_{i}(x, t)$, and let $\overline{\mathcal{V}}(t)=\left\{i \in \mathcal{V}: V_{i}(x(t), t)=\right.$ $V(x(t), t)\}$ be the set of indices where the maximum is reached at time $t$. Then

$$
D^{+} V(x(t), t)=\max _{i \in \overline{\mathcal{V}}(t)} \dot{V}_{i}(x(t), t) .
$$

The following lemma is a variant of Matrosov's theorem stated in [27], which will be very useful in analyzing uniformly asymptotically stable of the equilibrium of a nonautonomous system.

Lemma 2 (Matrosov's theorem). Given the system

$$
\dot{x}=f(t, x),
$$

where $f(t, 0)=0$ and $f(t, x)$ is piecewise continuous in $t$ and locally Lipschitz in $x$. Let $V(t, x)$ and $\psi(t, x)$ be continuously differentiable functions on domain $\left[t_{0}, \infty\right) \times \mathcal{D}$ and satisfy the following conditions:

1) $V(t, x)$ is positive definite and decrescent.

2) $\dot{V}(t, x) \leq W(x) \leq 0$, where $W(x)$ is continuous.

3) $|\psi(t, x)|$ is bounded.

4) The function $\dot{\psi}(t, x)$ is continuous in both arguments and $\dot{\psi}(t, x)=g(x, \chi(t))$, where $g$ is continuous in both of its arguments, $\chi(t)$ is also continuous and bounded.
5) There exists a class $\mathcal{K}$ function $\mu$ such that $|\dot{\psi}(t, x)| \geq$ $\mu(\|x\|), \forall x \in \mathcal{M}$, where $\mathcal{M}=\{x \mid W(x)=0\}$.

Then, the equilibrium of (4) is uniformly asymptotically stable on $\mathcal{D}$.

\section{PROBlem Formulation}

Suppose that there are $n$ agents in the group, labelled by $\mathcal{V}=\{1,2, \ldots, n\}$. The system dynamics of the agents are described by the Lagrangian equations

$$
M_{i}\left(q_{i}\right) \ddot{q}_{i}+C_{i}\left(q_{i}, \dot{q}_{i}\right) \dot{q}_{i}=\tau_{i}, \quad i=1,2, \ldots, n,
$$

where $q_{i} \in \mathbb{R}^{m}$ is the vector of generalized coordinates, $M_{i}\left(q_{i}\right) \in \mathbb{R}^{m \times m}$ is the $m \times m$ inertia (symmetric) matrix, $C_{i}\left(q_{i}, \dot{q}_{i}\right) \dot{q}_{i}$ is the Coriolis and centrifugal terms, and $\tau_{i} \in$ $\mathbb{R}^{m}$ is the control force. The dynamics of a Lagrangian system satisfies the following properties [28]:

1. $M_{i}\left(q_{i}\right)$ is positive definite and is bounded for any $q_{i} \in$ $\mathbb{R}^{m}$.

2. $\dot{M}_{i}\left(q_{i}\right)-2 C_{i}\left(q_{i}, \dot{q}_{i}\right)$ is skew symmetric.

3. $C_{i}\left(q_{i}, \dot{q}_{i}\right)$ is bounded with respect to $q_{i}$ and linearly bounded with respect to $\dot{q}_{i}$. More specifically, there is positive constant $k_{C}$ such that $\left\|C_{i}\left(q_{i}, \dot{q}_{i}\right)\right\| \leq k_{C}\left\|\dot{q}_{i}\right\|$.

We consider the set target aggregation problem for a group of Lagrangian systems. Each agent $i \in \mathcal{V}$ observes its own target set $X_{i}$. The objective is to ensure that the generalized coordinate derivatives of all the agents converge to zero and their generalized coordinates achieve agreement, while the destination of each agent is constrained by its target set. At each time, we assume that each agent observes the boundary points of its target set and obtain the relative distance information between the target set and itself. Also, the state information of each agent are exchanged by equipping each agent with simple and cheap communication unit. In the following, we give some necessary assumptions on set $X_{i}$, $i \in \mathcal{V}$ and define the set target aggregation problem.

Assumption 3.1. $X_{1}, X_{2}, \ldots, X_{n}$ are closed convex sets.

Assumption 3.2. $X_{0}=\bigcap_{i=1}^{n} X_{i}$ is nonempty and bounded.

Definition 1. Multi-agent system (5) is said to achieve set target aggregation if

1) $\lim _{t \rightarrow \infty}\left\|q_{i}(t)\right\|_{X_{0}}=0, \quad \forall i \in \mathcal{V}$,

2) $\lim _{t \rightarrow \infty}\left(q_{i}(t)-q_{j}(t)\right)=0, \quad \forall i, j \in \mathcal{V}$,

3) $\lim _{t \rightarrow \infty} \dot{q}_{i}(t)=0, \quad \forall i \in \mathcal{V}$.

Remark 1. In fact, this set target aggregation problem under convexity assumptions was a classical problem in optimization, where projected consensus algorithm was a standard solution [29]. This algorithm was then generalized to distributed versions via consensus dynamics in [30], [31]. However, all these existing algorithms are designed for agents with first-order dynamics, and are therefore not applicable to the problem studied in the current paper. 


\section{FiXED COMMUNiCATION TOPOLOGY}

In this section, the communication topology is assumed to be fixed. The following target aggregation algorithm is proposed for all $i \in \mathcal{V}$,

$$
\tau_{i}=-k \dot{q}_{i}-\sum_{j \in \mathcal{N}_{i}} a_{i j}\left(q_{i}-q_{j}\right)-\left(q_{i}-P_{X_{i}}\left(q_{i}\right)\right),
$$

where $k>0$ denotes generalized coordinate derivative damping, $a_{i j}$ is $(i, j)$ th entry of $\mathcal{A}$ associated with $\mathcal{G}$ defined in Section II-B. Note that $a_{i j}$ is a positive constant if $(j, i) \in$ $\mathcal{E}$ and $a_{i j}=0$ otherwise.

Theorem 1. Suppose Assumptions 3.1 and 3.2 hold. The multi-agent system (5) with (6) achieves set target aggregation in the sense of Definition 1 if the fixed communication topology $\mathcal{G}$ is connected.

Proof: In what follows, we will use Lemma 2 to prove Theorem 1. Consider the following Lyapunov function:

$$
\begin{aligned}
V= & \frac{1}{2} \sum_{i=1}^{n} \dot{q}_{i}^{\mathrm{T}} M_{i}\left(q_{i}\right) \dot{q}_{i}+\frac{1}{2} \sum_{i=1}^{n}\left\|q_{i}-P_{X_{i}}\left(q_{i}\right)\right\|^{2} \\
& +\frac{1}{4} \sum_{i=1}^{n} \sum_{j \in \mathcal{N}_{i}} a_{i j}\left\|q_{i}-q_{j}\right\|^{2} .
\end{aligned}
$$

Let $\widetilde{q}$ denote a column stack vector of all $q_{i}-q_{j}$, where $i<j$ and $a_{i j} \neq 0$. It then follows that $V$ is positive definite and decrescent with respect to $\widetilde{q}, q_{i}-P_{\mathcal{X}_{i}}\left(q_{i}\right), i \in \mathcal{V}$, and $\dot{q}_{i}$, $i \in \mathcal{V}$ since the communication graph $\mathcal{G}$ is connected. This verifies the first condition of Lemma 2. The derivative of $V$ along (5) with (6) is

$$
\begin{aligned}
\dot{V}= & \sum_{i=1}^{n} \dot{q}_{i}^{\mathrm{T}}\left(\frac{1}{2} \dot{M}_{i}\left(q_{i}\right) \dot{q}_{i}+M_{i}\left(q_{i}\right) \ddot{q}_{i}\right)+\sum_{i=1}^{n} \dot{q}_{i}^{\mathrm{T}}\left(q_{i}-P_{X_{i}}\left(q_{i}\right)\right) \\
& +\frac{1}{2} \sum_{i=1}^{n} \sum_{j \in \mathcal{N}_{i}} a_{i j}\left(q_{i}-q_{j}\right)^{\mathrm{T}}\left(\dot{q}_{i}-\dot{q}_{j}\right) \\
= & \sum_{i=1}^{n} \dot{q}_{i}^{\mathrm{T}}\left(-k \dot{q}_{i}-\left(q_{i}-P_{\mathcal{X}_{i}}\left(q_{i}\right)\right)-\sum_{j \in \mathcal{N}_{i}} a_{i j}\left(q_{i}-q_{j}\right)\right) \\
& +\sum_{i=1}^{n} \dot{q}_{i}^{\mathrm{T}}\left(q_{i}-P_{X_{i}}\left(q_{i}\right)\right)+\sum_{i=1}^{n} \dot{q}_{i}^{\mathrm{T}} \sum_{j \in \mathcal{N}_{i}} a_{i j}\left(q_{i}-q_{j}\right) \\
= & -\sum_{i=1}^{n} k \dot{q}_{i}^{\mathrm{T}} \dot{q}_{i}=: W \leq 0,
\end{aligned}
$$

where we have used (1) to derive the first equality, the fact that $a_{i j}=a_{j i}$ and the second property of Lagrangian dynamics to derive the second equality. The above inequality verifies the second condition of Lemma 2 .

Pick any $q_{0} \in \mathcal{X}_{0}$. Such a $q_{0}$ exists due to the fact that $\mathcal{X}_{0}$ is nonempty and bounded. Define $\psi=\sum_{i=1}^{n} \dot{q}_{i}^{\mathrm{T}} M_{i}\left(q_{i}\right)\left(q_{i}-\right.$ $\left.q_{0}\right)$. It follows that $q_{i}, \dot{q}_{i}$, for all $i \in \mathcal{V}$ are bounded from the first property of Lagrangian dynamics and the fact that $\dot{V} \leq 0$. Combining with the fact that $q_{0}$ is bounded, we know that $|\psi|$ is bounded. This verifies the third condition of Lemma 2. We then get that the derivative of $\psi$ is

$$
\begin{aligned}
\dot{\psi}= & \sum_{i=1}^{n} \ddot{q}_{i}^{\mathrm{T}} M_{i}\left(q_{i}\right)\left(q_{i}-q_{0}\right)+\sum_{i=1}^{n} \dot{q}_{i}^{\mathrm{T}} \dot{M}_{i}\left(q_{i}\right)\left(q_{i}-q_{0}\right) \\
& +\sum_{i=1}^{n} \dot{q}_{i}^{\mathrm{T}} M_{i}\left(q_{i}\right) \dot{q}_{i} \\
= & -\sum_{i=1}^{n} \dot{q}_{i}^{\mathrm{T}} C_{i}^{\mathrm{T}}\left(q_{i}\right)\left(q_{i}-q_{0}\right)-\sum_{i=1}^{n} k \dot{q}_{i}^{\mathrm{T}}\left(q_{i}-q_{0}\right) \\
& -\sum_{i=1}^{n}\left(q_{i}-q_{0}\right)^{\mathrm{T}} \sum_{j \in \mathcal{N}_{i}} a_{i j}\left(q_{i}-q_{j}\right) \\
& -\sum_{i=1}^{n}\left(q_{i}-P_{\mathcal{X}_{i}}\left(q_{i}\right)\right)^{\mathrm{T}}\left(q_{i}-q_{0}\right) \\
& +\sum_{i=1}^{n} \dot{q}_{i}^{\mathrm{T}} \dot{M}_{i}\left(q_{i}\right)\left(q_{i}-q_{0}\right)+\sum_{i=1}^{n} \dot{q}_{i}^{\mathrm{T}} M_{i}\left(q_{i}\right) \dot{q}_{i} .
\end{aligned}
$$

It is trivial to see that the form of $\dot{\psi}$ satisfies the fourth condition of Lemma 2 based on the facts that $q_{0}$ is a constant and $P_{X_{i}}\left(q_{i}\right)$ is a function of $q_{i}$. It follows that on the set $\mathcal{M}=\left\{q_{i} \in \mathbb{R}^{m}, \dot{q}_{i} \in \mathbb{R}^{m}, \forall i \in \mathcal{V} \mid \dot{q}_{i}=0, \forall i \in \mathcal{V}\right\}, \dot{\psi}$ becomes

$$
\begin{aligned}
\dot{\psi}= & -\sum_{i=1}^{n}\left(q_{i}-q_{0}\right)^{\mathrm{T}} \sum_{j \in \mathcal{N}_{i}} a_{i j}\left(q_{i}-q_{j}\right) \\
& -\sum_{i=1}^{n}\left(q_{i}-P_{X_{i}}\left(q_{i}\right)\right)^{\mathrm{T}}\left(q_{i}-q_{0}\right) .
\end{aligned}
$$

We know that $\sum_{i=1}^{n}\left(q_{i}-q_{0}\right)^{\mathrm{T}} \sum_{j \in \mathcal{N}_{i}} a_{i j}\left(q_{i}-q_{j}\right)$ $=\frac{1}{2} \sum_{i=1}^{n} \sum_{j \in \mathcal{N}_{i}} a_{i j}\left\|q_{i}-q_{j}\right\|^{2} \geq 0$ by noting that $a_{i j}=a_{j i}$. Also, we know from (2) that for all $i \in \mathcal{V},\left(P_{X_{i}}\left(q_{i}\right)-\right.$ $\left.q_{0}\right)^{\mathrm{T}}\left(q_{i}-P_{\mathcal{X}_{i}}\left(q_{i}\right)\right) \geq 0$. It then follows that

$$
\begin{aligned}
\left(q_{i}-q_{0}\right)^{\mathrm{T}}\left(q_{i}-P_{X_{i}}\left(q_{i}\right)\right)= & \left\|q_{i}-P_{X_{i}}\left(q_{i}\right)\right\|^{2} \\
& +\left(P_{X_{i}}\left(q_{i}\right)-q_{0}\right)^{\mathrm{T}}\left(q_{i}-P_{X_{i}}\left(q_{i}\right)\right) \\
\geq & \left\|q_{i}-P_{X_{i}}\left(q_{i}\right)\right\|^{2} .
\end{aligned}
$$

This shows that on the set $\mathcal{M}$,

$$
\dot{\psi} \leq-\frac{1}{2} \sum_{i=1}^{n} \sum_{j \in \mathcal{N}_{i}} a_{i j}\left\|q_{i}-q_{j}\right\|^{2}-\sum_{i=1}^{n}\left\|q_{i}-P_{X_{i}}\left(q_{i}\right)\right\|^{2} .
$$

Therefore, $|\dot{\psi}|$ is positive definite with respect to $\widetilde{q}$ and $q_{i}-P_{X_{i}}\left(q_{i}\right), i \in \mathcal{V}$. This verifies the last condition of Lemma 2. It thus follows from Lemma 2 that $\lim _{t \rightarrow \infty} \dot{q}_{i}(t)=$ $0, \lim _{t \rightarrow \infty}\left(q_{i}(t)-P_{X_{i}}\left(q_{i}(t)\right)\right)=0, \forall i \in \mathcal{V}$, and $\lim _{t \rightarrow \infty} \widetilde{q}(t)=0$. Therefore, we know that $\lim _{t \rightarrow \infty}\left(q_{i}(t)-\right.$ $\left.q_{j}(t)\right)=0, \forall i, j \in \mathcal{V}$ from the fact that $\mathcal{G}$ is connected. It then follows that for all $i \in \mathcal{V}$ and $l \in \mathcal{V}$

$$
\begin{aligned}
\left\|q_{i}-P_{X_{l}}\left(q_{i}\right)\right\| \leq & \left\|q_{i}-q_{l}\right\|+\left\|q_{l}-P_{X_{l}}\left(q_{l}\right)\right\| \\
& +\left\|P_{X_{l}}\left(q_{l}\right)-P_{X_{l}}\left(q_{i}\right)\right\| \\
\leq & 2\left\|q_{i}-q_{l}\right\|+\left\|q_{l}-P_{X_{l}}\left(q_{l}\right)\right\|,
\end{aligned}
$$

where we have used (3). This implies that $\lim _{t \rightarrow \infty}\left(q_{i}(t)-\right.$ $\left.P_{X_{l}}\left(q_{i}(t)\right)\right)=0, \forall i \in \mathcal{V}$ and $l \in \mathcal{V}$. Therefore, $\lim _{t \rightarrow \infty}\left\|q_{i}(t)\right\|_{X_{0}}=0, \forall i \in \mathcal{V}$. This shows that set target aggregation is achieved in the sense of Definition 1. 


\section{SWITCHING COMMUNiCATION TOPOLOGY}

One issue of introducing the communication unit is the possible communication link failure. Such issue becomes even more important when we consider the real applications including controlling multiple autonomous vehicles in the environments with limited power. Therefore, it is necessary to consider the case of switching communication topology. We associate the switching communication topology with a timevarying graph $\mathcal{G}_{\sigma(t)}=\left(\mathcal{V}, \mathcal{E}_{\sigma(t)}\right)$, where $\sigma:\left[t_{0},+\infty\right) \rightarrow \mathcal{P}$ is a piecewise constant function and $\mathcal{P}$ is finite set of all possible graphs. $\mathcal{G}_{\sigma(t)}$ remains constant for $t \in\left[t_{l}, t_{l+1}\right)$, $l=0,1, \ldots$ and switches at $t=t_{l}, l=1, \ldots$ In addition, we assume that $\inf _{l}\left(t_{l+1}-t_{l}\right) \geq \tau_{d}>0, l=1, \ldots$, where $\tau_{d}$ is a constant and this dwell time assumption is extensively used in the analysis of switched systems [32]. The joint graph of $\mathcal{G}_{\sigma(t)}$ during time interval $\left[t_{1}, t_{2}\right)$ is defined by $\mathcal{G}\left(\left[t_{1}, t_{2}\right)\right)=\bigcup_{t \in\left[t_{1}, t_{2}\right)} \mathcal{G}(t)=\left(\mathcal{V}, \bigcup_{t \in\left[t_{1}, t_{2}\right)} \mathcal{E}(t)\right)$. Moreover, $j$ is a neighbor of $i$ at time $t$ when $(j, i) \in \mathcal{E}_{\sigma(t)}$, and $\mathcal{N}(\sigma(t))$ represents the set of agent $i$ 's neighbors at time $t$.

Definition 2. $\mathcal{G}_{\sigma(t)}$ is uniformly jointly connected if there exists a constant $T>0$ such that $\mathcal{G}([t, t+T))$ is connected for any $t \geq t_{0}$.

The existence of the switching communication topology complicates the problem significantly. In order to simplify the problem, we assume that the exact information of system dynamical parameters are available and propose the following algorithm

$$
\tau_{i}=C_{i}\left(q_{i}, \dot{q}_{i}\right) \dot{q}_{i}+M_{i}\left(q_{i}\right) u_{i}, \quad \forall i \in \mathcal{V},
$$

where

$u_{i}=-k \dot{q}_{i}-\sum_{j \in \mathcal{N}_{i}(\sigma(t))} a_{i j}(\sigma(t))\left(q_{i}-q_{j}\right)-\left(q_{i}-P_{X_{i}}\left(q_{i}\right)\right)$,

$k>0$ denotes generalized coordinate derivative damping, and $a_{i j}(p)$ is the weight of arc $(j, i)$ associated with graph $\mathcal{G}_{p}$, for all $p \in \mathcal{P}$.

By introducing (9) into (5), we have

$$
\ddot{q}_{i}=-k \dot{q}_{i}-\sum_{j \in \mathcal{N}_{i}(\sigma(t))} a_{i j}(\sigma(t))\left(q_{i}-q_{j}\right)-\left(q_{i}-P_{X_{i}}\left(q_{i}\right)\right) .
$$

We next focus on the closed-loop system (10).

Lemma 3. Suppose that Assumptions 3.1 and 3.2 hold and choose $k$ large enough. The states of the multi-agent system (5) with (9) achieve local target aggregation, i.e., $\lim _{t \rightarrow \infty}\left\|q_{i}(t)\right\|_{X_{i}}=0$, and $\lim _{t \rightarrow \infty} \dot{q}_{i}(t)=0$, for all $i \in \mathcal{V}$.

Proof: By picking any $q_{0} \in X_{0}$, we propose the following Lyapunov function:

$$
\begin{aligned}
V= & \frac{1}{2} \sum_{i=1}^{n} \dot{q}_{i}^{\mathrm{T}} \dot{q}_{i}+\sum_{i=1}^{n}\left(q_{i}-q_{0}\right)^{\mathrm{T}} \dot{q}_{i}+\frac{k}{2} \sum_{i=1}^{n}\left\|q_{i}-q_{0}\right\|^{2} \\
& +\frac{1}{2} \sum_{i=1}^{n}\left\|q_{i}-P_{X_{i}}\left(q_{i}\right)\right\|^{2},
\end{aligned}
$$

where we choose $k>1$ to guarantee $V$ is positive definite. The derivative of $V$ along (10) is

$$
\begin{aligned}
& \dot{V}=\sum_{i=1}^{n} \dot{q}_{i}^{\mathrm{T}}\left(-k \dot{q}_{i}-\sum_{j \in \mathcal{N}_{i}(\sigma(t))} a_{i j}(\sigma(t))\left(q_{i}-q_{j}\right)\right. \\
& -\left(q_{i}-P_{X_{i}}\left(q_{i}\right)\right)+\sum_{i=1}^{n}\left(q_{i}-q_{0}\right)^{\mathrm{T}}\left(-k \dot{q}_{i}\right. \\
& \left.-\sum_{j \in \mathcal{N}_{i}(\sigma(t))} a_{i j}(\sigma(t))\left(q_{i}-q_{j}\right)-\left(q_{i}-P_{X_{i}}\left(q_{i}\right)\right)\right) \\
& +\sum_{i=1}^{n}\left\|\dot{q}_{i}\right\|^{2}+k \sum_{i=1}^{n}\left(q_{i}-q_{0}\right)^{\mathrm{T}} \dot{q}_{i}+\sum_{i=1}^{n} \dot{q}_{i}^{\mathrm{T}}\left(q_{i}-P_{X_{i}}\left(q_{i}\right)\right) \\
& =-(k-1) \sum_{i=1}^{n}\left\|\dot{q}_{i}\right\|^{2}-\sum_{i=1}^{n} \dot{q}_{i}^{\mathrm{T}} \sum_{j \in \mathcal{N}_{i}(\sigma(t))} a_{i j}(\sigma(t)) \\
& \times\left(q_{i}-q_{j}\right)-\sum_{i=1}^{n}\left(q_{i}-q_{0}\right)^{\mathrm{T}}\left(q_{i}-P_{X_{i}}\left(q_{i}\right)\right) \\
& -\sum_{i=1}^{n}\left(q_{i}-q_{0}\right)^{\mathrm{T}} \sum_{j \in \mathcal{N}_{i}(\sigma(t))} a_{i j}(\sigma(t))\left(q_{i}-q_{j}\right) \\
& \leq-\left[\begin{array}{ll}
\bar{q} & \dot{q}
\end{array}\right]\left[\begin{array}{cc}
\mathcal{L}_{\sigma} & \frac{\mathcal{L}_{\sigma}}{2} \\
\frac{\mathcal{L}_{\sigma}}{2} & (k-2) I_{n}
\end{array}\right]\left[\begin{array}{c}
\bar{q} \\
\dot{q}
\end{array}\right] \\
& -\sum_{i=1}^{n}\left\|q_{i}\right\|_{X_{i}}^{2}-\sum_{i=1}^{n}\left\|\dot{q}_{i}\right\|^{2}
\end{aligned}
$$

where $\bar{q}_{i}=q_{i}-q_{0}, \bar{q}=\left[\bar{q}_{1}^{\mathrm{T}}, \bar{q}_{2}^{\mathrm{T}}, \ldots, \bar{q}_{n}^{\mathrm{T}}\right]^{\mathrm{T}}, q=$ $\left[q_{1}^{\mathrm{T}}, q_{2}^{\mathrm{T}}, \ldots, q_{n}^{\mathrm{T}}\right]^{\mathrm{T}}, \mathcal{L}_{\sigma}$ is a weighted Laplacian matrix associated with $\mathcal{G}_{\sigma}$ defined in Section II-B, and we have used the fact that $\left(q_{i}-q_{0}\right)^{\mathrm{T}}\left(q_{i}-P_{X_{i}}\left(q_{i}\right)\right) \geq\left\|q_{i}-P_{X_{i}}\left(q_{i}\right)\right\|^{2}$. We know that all the eigenvalues of $\mathcal{L}_{p}$ are non-positive and real, for all $p \in \mathcal{P}$. Therefore, if $k$ is chosen such that $k>2+$ $\frac{1}{4} \max _{p \in \mathcal{P}}\left\{\lambda_{\max }\left(\mathcal{L}_{p}\right)\right\}$, or equivalent, $k>2+\frac{(n-1) a^{*}}{2}$, we can show that $\left[\begin{array}{cc}\mathcal{L}_{p} & \frac{\mathcal{L}_{p}}{2} \\ \frac{\mathcal{L}_{p}}{2} & (k-2) I_{n}\end{array}\right]$ is positive semi-definite, for all $p \in \mathcal{P}$, where $a^{*}=\max _{p \in \mathcal{P}} \max _{i, j \in \mathcal{V}} a_{i j}(p)$. Under this condition, it then follows that

$$
\dot{V} \leq-\sum_{i=1}^{n}\left\|q_{i}\right\|_{X_{i}}^{2}-\sum_{i=1}^{n}\left\|\dot{q}_{i}\right\|^{2} \leq 0
$$

Therefore, $q_{i}$ and $\dot{q}_{i}, \forall i \in \mathcal{V}$ are bounded. We also know that (11) implies that

$$
\int_{t_{0}}^{\infty}\left(\sum_{i=1}^{n}\left\|q_{i}(t)\right\|_{X_{i}}^{2}+\sum_{i=1}^{n}\left\|\dot{q}_{i}(t)\right\|^{2}\right) \mathrm{d} t
$$

is bounded by $V\left(t_{0}\right)$. In addition, it follows that $\frac{\mathrm{d}}{\mathrm{d} t}\left(\sum_{i=1}^{n}\left\|q_{i}(t)\right\|_{X_{i}}^{2}+\sum_{i=1}^{n}\left\|\dot{q}_{i}(t)\right\|^{2}\right)=2 \sum_{i=1}^{n}$ $\left(\left(q_{i}-P_{X_{i}}\left(q_{i}\right)\right)^{\mathrm{T}} \dot{q}_{i}+\dot{q}_{i}^{\mathrm{T}} \ddot{q}_{i}\right)$. Therefore, from (10) and the facts that $q_{i}$ and $\dot{q}_{i}, \forall i \in \mathcal{V}$ are bounded, we know that $\frac{\mathrm{d}}{\mathrm{d} t}\left(\sum_{i=1}^{n}\left\|q_{i}(t)\right\|_{X_{i}}^{2}+\sum_{i=1}^{n}\left\|\dot{q}_{i}(t)\right\|^{2}\right)$ is bounded, $\forall t \geq$ $t_{0}$. Then, based on Barbalat's lemma, we can show that $\sum_{i=1}^{n}\left\|q_{i}(t)\right\|_{X_{i}}^{2}+\sum_{i=1}^{n}\left\|\dot{q}_{i}(t)\right\|^{2} \rightarrow 0$, as $t \rightarrow \infty$. Therefore, $\lim _{t \rightarrow \infty}\left\|q_{i}(t)\right\|_{X_{i}}=0$, and $\lim _{t \rightarrow \infty} \dot{q}_{i}(t)=0$, for all $i \in \mathcal{V}$. 
Next, we define $x_{i}=q_{i}, x_{n+i}=q_{i}+\dot{q}_{i}$, for all $i \in \mathcal{V}$. After some manipulations, (10) can be written as

$$
\begin{gathered}
\dot{x}_{i}=-\left(x_{i}-x_{n+i}\right), \\
\dot{x}_{n+i}=-\sum_{j \in \mathcal{N}_{i}(\sigma(t))} a_{i j}(\sigma(t))\left(x_{n+i}-x_{n+j}\right)+\delta_{i},
\end{gathered}
$$

where $i \in \mathcal{V}$, and $\delta_{i}=(1-k) \dot{q}_{i}+\sum_{j \in \mathcal{N}_{i}(\sigma(t))} a_{i j}(\sigma(t))\left(\dot{q}_{i}-\right.$ $\left.\dot{q}_{j}\right)-\left(q_{i}-P_{\mathcal{X}_{i}}\left(q_{i}\right)\right)$, for all $i \in \mathcal{V}$. Note that Lemma 3 implies that $\lim _{t \rightarrow \infty} \delta_{i}(t)=0$, for all $i \in \mathcal{V}$. We next present the main result of this section.

Theorem 2. Suppose that Assumptions 3.1 and 3.2 hold and choose $k$ large enough. The multi-agent system (5) with (9) achieves set target aggregation in the sense of Definition 1 if the communication topology $\mathcal{G}_{\sigma(t)}$ is uniformly jointly connected.

Proof: Consider system (12) as a new multi-agent system with node set $\overline{\mathcal{V}}=\{1,2, \ldots, 2 n\}$. We associate this system with a new graph $\overline{\mathcal{G}}_{\sigma(t)}=\left(\overline{\mathcal{V}}, \overline{\mathcal{E}}_{\sigma(t)}\right)$, the corresponding neighbor set $\overline{\mathcal{N}}_{i}(\sigma(t))$ and the adjacency matrix $\bar{A}_{\sigma(t)}$. Note that the connections and weights for agents $\{n+1, n+$ $2, \ldots, 2 n\}$ are defined by $\mathcal{E}_{\sigma(t)}$ and $A_{\sigma(t)}$. In addition, there exists persistent arc $a_{i(i+n)}(t)=1$, for all $i=1,2, \ldots, n$ and all $t \geq t_{0}$. It is not hard to see that $\overline{\mathcal{G}}_{\sigma(t)}$ is uniformly jointly quasi-strongly connected with the uniform constant $T$, where $\overline{\mathcal{G}}_{\sigma(t)}$ is said to be uniformly jointly quasi-strongly connected if there exists a node $i \in \overline{\mathcal{V}}$ and a constant $T>0$ such that there exists a directed path from $i$ to any other node for $\overline{\mathcal{G}}([t, t+T)), \forall t \geq t_{0}$.

Then, based on Proposition 4.10 of [33] and the fact that $\lim _{t \rightarrow \infty} \delta_{i}(t)=0$, for all $i \in \mathcal{V}$, we know that $\lim _{t \rightarrow \infty}\left(q_{i}(t)-q_{j}(t)\right)=0, \forall i, j \in \mathcal{V}$. Following (8) in the proof of Theorem 1, we know that $\lim _{t \rightarrow \infty}\left\|q_{i}(t)\right\|_{\mathcal{X}_{0}}=0$, $\forall i \in \mathcal{V}$. This shows that set target aggregation is achieved in the sense of Definition 1.

\section{Simulation Results}

In this section, we use numerical simulations to validate the effectiveness of the theoretical results obtained in this paper. We assume that there are four agents $(n=4)$ in the group. The system dynamics of the followers are given by the following two-link manipulators [20], [28],

$$
\begin{array}{r}
{\left[\begin{array}{ll}
M_{11, i} & M_{12, i} \\
M_{21, i} & M_{22, i}
\end{array}\right]\left[\begin{array}{l}
\ddot{q}_{i x} \\
\ddot{q}_{i y}
\end{array}\right]+} \\
=\left[\begin{array}{ll}
C_{11, i} & C_{12, i} \\
C_{21, i} & C_{22, i}
\end{array}\right]\left[\begin{array}{c}
\dot{q}_{i x} \\
\dot{q}_{i y}
\end{array}\right] \\
=\left[\begin{array}{c}
\tau_{i x} \\
\tau_{i y}
\end{array}\right], i=1,2,3,4,
\end{array}
$$

where $M_{11, i}=\theta_{1 i}+2 \theta_{2 i} \cos q_{i y}, M_{12, i}=M_{21, i}=\theta_{3 i}+$ $\theta_{2 i} \cos q_{i y}, M_{22, i}=\theta_{3 i}, C_{11, i}=-\theta_{2 i} \sin q_{i y} \dot{q}_{i y}, C_{12, i}=$ $-\theta_{2 i} \sin q_{i y}\left(\dot{q}_{i x}+\dot{q}_{i y}\right), C_{21, i}=\theta_{2 i} \sin q_{i y} \dot{q}_{i x}, C_{22, i}=0$. We choose $\theta_{1 i}=1.301, \theta_{2 i}=0.256, \theta_{3 i}=0.096, i=1,2,3,4$. The available local sets of all the agents are rectangles given by $X_{1}=[-0.5,1] \times[-0.5,1], X_{2}=[0.5,-1] \times[0.5,-1]$, $X_{3}=[-0.5,1] \times[-0.5,1]$, and $X_{4}=[0.5,-1] \times[0.5,-1]$. The initial states of the agents are given by $q_{1}(0)=[-2,2]^{\mathrm{T}}$,

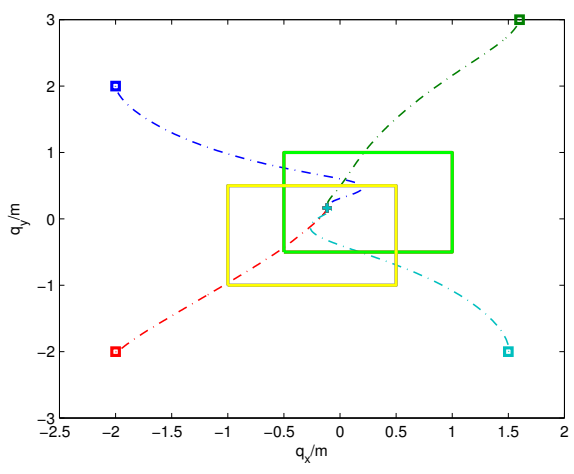

Fig. 1. The trajectories of the generalized coordinates of the agents using (6) for (5) under a fixed communication topology. The squares and the cross denote, respectively, the initial and final generalized coordinates of the agents. The big rectangles denote the available local sets for each agent.

$q_{2}(0)=[1.6,3]^{\mathrm{T}}, q_{3}(0)=[-2,-2]^{\mathrm{T}}, q_{4}(0)=[1.5,-2]^{\mathrm{T}}$, $\dot{q}_{1}(0)=[-0.1,0.1]^{\mathrm{T}}, \quad \dot{q}_{2}(0)=[0.2,-0.2]^{\mathrm{T}}, \dot{q}_{3}(0)=$ $[0.7,-0.7]^{\mathrm{T}}$, and $\dot{q}_{4}(0)=[0.4,-0.4]^{\mathrm{T}}$.

For the case of the fixed communication topology, the control parameter is chosen by $k=1$. The weighted adjacency matrix $\mathcal{A}$ of the generalized coordinates associated with $\mathcal{G}$ is chosen to be $\mathcal{A}=\left[\begin{array}{llll}0 & 2 & 0 & 4 \\ 2 & 0 & 1 & 0 \\ 0 & 1 & 0 & 1 \\ 4 & 0 & 1 & 0\end{array}\right]$. Using (6) for (5), the trajectories of the generalized coordinates of the agents are shown in Fig. 1. We see that set target aggregation is achieved.

The control parameter is chosen by $k=5$. The weighted adjacency matrix $\mathcal{A}(t)$ of the generalized coordinates associated with $\mathcal{G}(t)$ switches between $\mathcal{A}_{1}$ and $\mathcal{A}_{2}$ at time instants $t_{\varepsilon}=5 \varepsilon, \varepsilon=0,1, \ldots$, where $\mathcal{A}_{1}=\left[\begin{array}{llll}0 & 2 & 0 & 0 \\ 2 & 0 & 0 & 0 \\ 0 & 0 & 0 & 1 \\ 0 & 0 & 1 & 0\end{array}\right]$, and $\mathcal{A}_{2}=\left[\begin{array}{llll}0 & 0 & 0 & 4 \\ 0 & 0 & 1 & 0 \\ 0 & 1 & 0 & 0 \\ 4 & 0 & 0 & 0\end{array}\right]$. Using (9) for (5), the trajectories of the generalized coordinates of the agents are shown in Fig. 2. We see that set target aggregation is achieved even when the communication topology is switching.

\section{CONCLUSIONS}

In this paper, we studied set target aggregation of multiple mechanical systems. Each system is modelled by the Lagrangian dynamics. The objective is to achieve generalized coordinate derivative convergence and generalized coordinate targeted agreement for all the agents. By exchanging information with local neighbors, we proposed set target aggregation algorithms and showed that all the agents converge into the intersection of all the local target sets over both fixed and switching communication topologies. Simulation are given to validate the theoretical results. 


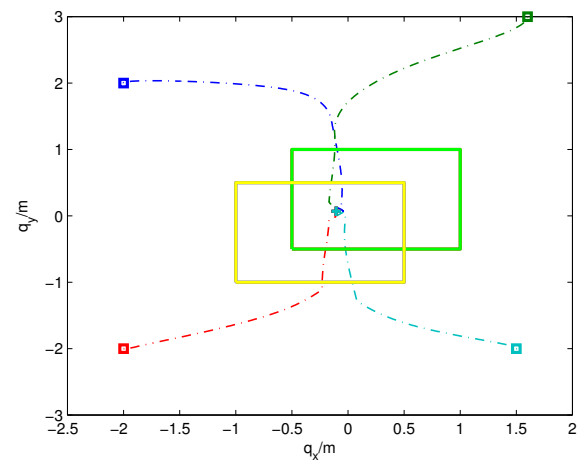

Fig. 2. The trajectories of the generalized coordinates of the agents using (9) for (5) under switching communication topologies. The squares and the cross denote, respectively, the initial and final generalized coordinates of the agents. The big rectangles denote the available local sets for each agent.

\section{REFERENCES}

[1] T. Vicsek, A. Czirok, E. B. Jacob, I. Cohen, and O. Schochet, "Novel type of phase transitions in a system of self-driven particles," Physical Review Letters, vol. 75, no. 6, pp. 1226-1229, 1995.

[2] A. Jadbabaie, J. Lin, and A. S. Morse, "Coordination of groups of mobile autonomous agents using nearest neighbor rules," IEEE Transactions on Automatic Control, vol. 48, no. 6, pp. 988-1001, 2003.

[3] Z. Lin, M. Broucke, and B. Francis, "Local control strategies for groups of mobile autonomous agents," IEEE Transactions on Automatic Control, vol. 49, no. 4, pp. 622-629, 2004.

[4] R. Olfati-Saber, J. A. Fax, and R. M. Murray, "Consensus and cooperation in networked multi-agent systems," Proceedings of the IEEE, vol. 95 , no. 1 , pp. $215-233,2007$.

[5] H. G. Tanner, A. Jadbabaie, and G. J. Pappas, "Flocking in fixed and switching networks," IEEE Transactions on Automatic Control, vol. 52, no. 5, pp. 863-868, 2007.

[6] G. Shi, Y. Hong, and K. H. Johansson, "Connectivity and set tracking of multi-agent systems guided by multiple moving leaders," IEEE Transactions on Automatic Control, vol. 57, no. 3, pp. 663-676, 2013.

[7] W. Ren and R. W. Beard, "Consensus seeking in multiagent systems under dynamically changing interaction topologies," IEEE Transactions on Automatic Control, vol. 50, no. 5, pp. 655-661, 2005.

[8] M. Cao, A. S. Morse, and B. D. O. Anderson, "Reaching a consensus in a dynamically changing environment: convergence rates, measurement delays, and asynchronous events," SIAM Journal of Control and Optimization, vol. 47, no. 2, pp. 601-623, 2008.

[9] Z. Lin, B. Francis, and M. Maggiore, "State agreement for continuoustime coupled nonlinear systems," SIAM Journal of Control and Optimization, vol. 46, no. 1, pp. 288-307, 2007.

[10] G. Shi and Y. Hong, "Global target aggregation and state agreement of nonlinear multi-agent systems with switching topologies," Automatica, vol. 45, no. 5, pp. 1165-1175, 2009.

[11] T. Yang, S. Roy, Y. Wan, and A. Saberi, "Constructing consensus controllers for networks with identical general linear agents," International Journal of Robust and Nonlinear Control, vol. 21, no. 11, pp. 1237-1256, 2011

[12] Z. Meng, Z. Lin, and W. Ren, "Robust cooperative tracking for multiple non-identical second-order nonlinear systems," Automatica, vol. 49, no. 8, pp. 2363-2372, 2013.

[13] S.-J. Chung and J.-J. E. Slotine, "Cooperative robot control and concurrent synchronization of Lagrangian systems," IEEE Transations on Robotics, vol. 25, no. 3, pp. 686-700, 2009.

[14] Y.-C. Liu and N. Chopra, "Controlled synchronization of heterogeneous robotic manipulators in the task space," IEEE Transactions on Robotics, vol. 28, no. 1, pp. 268-275, 2012.

[15] W. Dong, "Tracking control of multiple-wheeled mobile robots with limited information of a desired trajectory," IEEE Transactions on Robotics, vol. 28, no. 1, pp. 262-268, 2012.

[16] Z. Meng, D. V. Dimarogonas, and K. H. Johansson, "Leader-follower coordinated tracking of multiple heterogeneous Lagrange systems using continuous control," IEEE Transactions on Robotics, vol. 30 no. 3, pp. 739-745, 2014.

[17] W. Ren, "Distributed leaderless consensus algorithms for networked Euler-Lagrange systems," International Journal of Control, vol. 82, no. 11, pp. 2137-2149, 2009.

[18] D. V. Dimarogonas and K. J. Kyriakopoulos, "On the rendezvous problem for multiple nonholonomic agents," IEEE Transactions on Automatic Control, vol. 52, no. 5, pp. 916-922, 2007.

[19] S. Khoo, L. Xie, and Z. Man, "Robust finite-time consensus tracking algorithm for multirobot systems," IEEE/ASME Transcations on Mechatronics, vol. 14, no. 2, pp. 219-228, 2009.

[20] J. Mei, W. Ren, and G. Ma, "Distributed coordinated tracking with a dynamic leader for multiple Euler-Lagrange systems," IEEE Transcations on Automatic Control, vol. 56, no. 6, pp. 1415-1421, 2011

[21] G. Chen and F. L. Lewis, "Distributed adaptive tracking control for synchronization of unknown networked Lagrangian systems," IEEE Transcations on Systems, Man and Cybernetics-Part B: Cybernetics, vol. 41, no. 3, pp. 805-816, 2011.

[22] W. Dong, "On consensus algorithms of multiple uncertain mechanical systems with a reference trajectory," Automatica, vol. 47, no. 9, pp. 2023-2028, 2011.

[23] Z. Meng, Z. Lin, and W. Ren, "Leader-follower swarm tracking for networked Lagrange systems," Systems and Control Letters, vol. 61, no. 1, pp. 117-126, 2012.

[24] R. Haghighi and C. C. Cheah, "Multi-group coordination control for robot swarms," Automatica, vol. 48, no. 10, pp. 2526-2534, 2012.

[25] J.-P. Aubin, Viability Theory. Birkhauser Boton, Boston, 1991.

[26] J. M. Danskin, "The theory of max-min, with applications," SIAM Journal on Applied Mathematics, vol. 14, no. 4, pp. 641-664, 1966.

[27] B. Paden and R. Panja, "Globally asymptotically stable PD+ controller for robot manipulators," International Journal of Control, vol. 47, no. 6 , pp. 1697-1712, 1988.

[28] M. W. Spong, S. Hutchinson, and M. Vidyasagar, Robot Dynamics and Control. John Wiley \& Sons, Inc., 2006.

[29] L. G. Gubin, B. T. Polyak, and E. V. Raik, "The method of projections for finding the common point of convex sets," USSR Computational Mathematics and Mathematical Physics, vol. 7, no. 6, pp. 1211-1228, 1967.

[30] A. Nedic, A. Ozdaglar, and P. A. Parrilo, "Constrained consensus and optimization in multi-agent networks," IEEE Transactions on Automatic Control, vol. 55, no. 4, pp. 922-938, 2010.

[31] G. Shi, K. H. Johansson, and Y. Hong, "Reaching an optimal consensus: dynamical systems that compute intersections of convex sets," IEEE Transactions on Automatic Control, vol. 58, no. 3, pp. 610-622, 2013.

[32] D. Liberzon and A. S. Morse, "Basic problems in stability and design of switched systems," IEEE Control Systems Magazine, vol. 19, pp. $59-70,1999$.

[33] G. Shi and K. H. Johansson, "Robust consensus for continuous-time multi-agent dynamics," SIAM Journal on Control and Optimization, vol. 51 , no. 5, pp. 3673-3691, 2013 